\title{
Navigation Ground Augmentation System Based on a Wireless Sensor
}

\author{
https://doi.org/10.3991/ijoe.v14i08.9183 \\ Le Le \\ Changsha Aeronautical Vocational and Technical College, Changsha, Changsha \\ lele37491@163.com
}

\begin{abstract}
To improve the performance of navigation systems and expand their application, a navigation ground augmentation system platform was designed based on wireless sensor. The system included the signal augmentation generation and reception verification platforms. The enhanced signaltransmitting and -receiving parameters were controlled through two sets of visual host software. Meanwhile, the performance indexes of the navigation ground augmentation platform were tested. Finally, the received data was captured by MATLAB coding process and the navigable messages were tracked. Results showed that the system satisfied the design requirements and could effectively improve the performance of the navigation system.
\end{abstract}

Keywords-wireless sensor, navigation ground augmentation system, signal augmentation generation platform, signal augmentation reception verification platform

\section{Introduction}

Wireless sensor network (WSN) is the combination of sensors, computers, communication, and other scientific fields. WSN nodes communicate by radio and form the network according to the needs of organizations and then transmit information to each other for publishing to users. For some important information, each node can perform computing processing, storage, and wireless communication tasks. These nodes can sense the surrounding environment within a distance. The WSN formed through free networking is also a combination of information acquisition (sensing), transmission, and processing. WSN is a popular IT technology that has made a great impact on human life in the 21st century after the Internet. In 1999, US Business Week listed WSNs as one of the 21 most influential technologies in the 21st century. In 2003, MIT Technology Review listed WSN technology as one of the 10 new technologies predicted to change the world in future technology development. In the same year, American Business Week cited WSN as one of the four high-technology industries in the world.

Navigation is a means to guiding aerocraft, vehicles, and other carriers accurately along set routes toward destinations. With the development of navigation technology, traditional navigation technology becomes unable to meet the requirements. The re- 
quirements for navigation performance are also increasing. Thus, the need to develop a new generation of navigation technology that is more secure, efficient, and reliable is urgent. Modern navigation technologies are based on radio navigation devices and are constantly evolving, including users and navigation signal transmitters. Users can obtain location information by calculating the signals transmitted by one or more navigation signal transmitters. Some radio navigation devices can also provide users with speed and time for broadcasting. The global navigation satellite system (GNSS) is now widely used, and it is increasingly dependent on satellite navigation in all fields of the national economy. Meanwhile, some insufficiencies of satellite navigation have received increasing attention. In application fields, such as aircraft landing navigation, vehicle navigation in the city, and precision agriculture, ordinary receivers have been unable to meet the requirements for navigation accuracy, integrity, availability, and continuity. On the basis of availability, completeness, positioning accuracy, and continuity, most satellite navigation systems can still meet the needs in addition to continuity parameters, and some have significant shortcomings. Therefore, the navigation performance augmentation theory and technology, which can not only be compatible with existing navigation systems but also play a role in the future navigation system and the coexistence of the new and old navigation systems, should be studied on the basis of existing navigation system facilities.

The research on the navigation ground augmentation system is conducted based on the WSN, and the WSN and navigation technologies are further developed and applied.

\section{State of the art}

Kang et al. (2015) reported that the WSN is composed of numerous micro sensor nodes that are densely distributed in the monitoring area [1]. These micro sensor nodes have communication and computing power. The autonomous control network system is formed by micro wireless modes through wireless means. Fan et al. (2017) indicated that the main applications of WSNs are divided into three types, that is, cooperative perception, collection and processing of information of perceived objects in the network coverage area, and transmission to users [2]. Zeng et al. (2016) highlighted that WSNs are flexible, low cost, and easy to implement and are thus widely used in military, environmental monitoring, medical insurance, space exploration, and other applications [3].

Dobrev et al. (2017) indicated that two types of satellite navigation augmentation systems exist, namely, the star-based augmentation system (SBAS) and the groundbased augmentation system (GBAS). The augmentation system adopts multiple navigation augmentation technologies to improve the performance indicators of satellite navigation services. On the basis of the four parameters that reflect the navigation performance, the augmentation technology can be divided into four categories, namely, precision, integrity, continuity, and availability augmentation. The difference principle is used to enhance precision, the integrity monitoring principle is generally adopted to enhance integrity, while the continuity and availability augmentation tech- 
niques improve the navigation signal source [4]. Zhang et al. (2017) reported that major spaceflight circles compete seriously and several SBAS systems have been built around the world, such as the United States wide area augmentation system, the Russian system of differential correction and monitoring, and the European geostationary navigation overlap service. Aldoumani et al. (2016) stated that the GBAS mainly uses the difference algorithm to improve the navigation precision of the global positioning system (GPS) satellite. According to the signal integrity algorithm, the integrity information of the GPS signal is obtained at a certain time, and the integrity, availability, and continuity of the GPS signal are improved [6]. Kumar et al. (2017) reported that the continuous operation reference station system is a widely used GBAS and belongs to the products of advanced science and technology, such as navigation satellite positioning technology, modern digital communication technology, and rapidly developed computer network technology [7]. Zhuang et al. (2016) suggested that ground-based pseudolite augmentation technology is also a GBAS. Pseudolite can broadcast the same or similar signals as GNSS navigation signals, providing user positioning, navigation, and timing services [8].

The aforementioned studies mainly focus on WSNs and navigation augmentation systems. Therefore, on the basis of the research status, the present work focuses on the research on navigation ground augmentation system based on wireless sensor. First, WSN is introduced. Then, the wireless sensor is applied to the navigation augmentation system platform to optimize the function of the navigation augmentation system.

\section{Method}

\subsection{Node design of WSN}

Sensor node is an important part of a WSN. The performance of sensor nodes is directly related to the performance of the entire network. Thus, the node design of the sensor network is important in the design of the entire system. Generally, each sensor node must satisfy the requirements of communication distance, communication quality, anti-interference capability, and low power consumption. In an ad hoc network, sensor nodes must provide convenient networking and must be easy to control. In some applications, sensor nodes must also be portable. In the design and application of sensor nodes, the cost, volume, flexibility, extensibility, stability, and security of the system should be considered.

Wireless sensor nodes include the simulator interface and the sensor, wireless communication, and power supply modules. Figure 1 presents the sensor node structure diagram. The wireless communication module is the hardware base of the communication, and the sensor module is the key module in completing the function of sensor nodes. The energy consumption data of commonly used WSN nodes are shown in Table 1. 


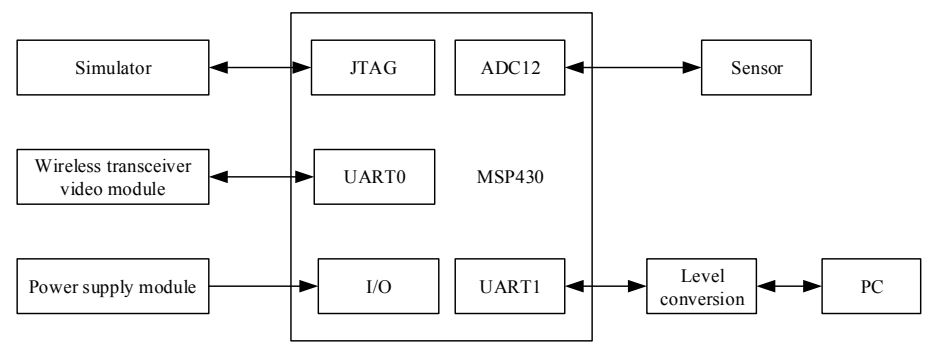

Fig. 1. Structure of sensor nodes

Table 1. Comparison of energy consumption of sensor nodes

\begin{tabular}{|l|c|c|c|}
\hline \multicolumn{1}{|c|}{ Operation } & Telos/Tmote & Mica2 & MicaZ \\
\hline Voltage & $1.8 \mathrm{~V}$ & $2.7 \mathrm{~V}$ & $2.7 \mathrm{~V}$ \\
\hline MCU (Microcontroller Unit) Active & $1.8 \mathrm{~mA}$ & $8.0 \mathrm{~mA}$ & $8.0 \mathrm{~mA}$ \\
\hline MCU Idle & $0.054 \mathrm{~mA}$ & $<2 \mathrm{~mA}$ & $<2 \mathrm{~mA}$ \\
\hline MCU + Radio RX & $21.8 \mathrm{~mA}$ & $16 \mathrm{~mA}$ & $27.7 \mathrm{~mA}$ \\
\hline MCU + Radio TX & $19.5 \mathrm{~mA}$ & $33 \mathrm{~mA}$ & $22 \mathrm{~mA}$ \\
\hline
\end{tabular}

\subsection{Ground augmentation signal design}

GPS system is currently the most widely used navigation system in the world. The navigation augmentation signal is based on the GPS satellite signal theory, such that the receiver can capture the enhanced signal with as little change as possible. The compatibility of navigation systems can be improved with the growth of navigation signal augmentation platform technologies, thereby improving their application potential.

In contrast to the GPS signal, the ground augmentation signal includes the navigation message D code and the C/A code, whereas the intermediate-frequency subcarrier is added. After spreading the D and C/A codes, the spread spectrum code is modulated with the intermediate-frequency subcarrier and then converted to the radio frequency (RF) carrier band through the second time-frequency change, such as 1575.42 MHz. The GPS signal is easily disturbed by the outside world; thus, the time slot can be controlled in the time domain to strengthen the performance of all aspects of the ground augmentation signal. The launch time and the length of the navigation augmentation signal can be defined. To utilize fully the frequency band resources, the multi medium-frequency subcarrier is used, the pseudocode and the navigation message are adapted for each subcarrier modulation, and the multiplex subcarrier is converted to the frequency band after the second time-frequency conversion when the signal is generated. The pseudocode rate of each subcarrier should be adjusted according to different accuracy requirements.

The augmentation signal should consider various future requirements and applications. In the ground augmentation system, we design a flexible and variable augmented navigation signal source in the ground augmentation system based on the naviga- 
tion signals from an ordinary GPS satellite. The ground augmentation system consists of multiple sets of signal generation equipment systems, and each device source signal is generated according to Equation (1).

$$
S^{i}(t)=\sum_{m}^{M^{i}} \sqrt{2 P_{m}^{i}} D^{i}(t) c^{i}(t) \sin \left(2 \pi\left(f_{c}+m \Delta f\right) t+\phi_{m}^{i}\right) g_{s}\left(\frac{t-\tau^{i}\left(\frac{\bmod \left(t, N T_{b}\right)}{T_{b}}\right)}{T_{b}}\right) .
$$

In the formula, $S^{i}$ is the signal launched by the $i$ th device, $t$ refers to time, ${ }^{i}$ is the signal transmitting power of the $m$ th subcarrier, $m^{i}$ denotes the number of subcarriers, $D^{i}$ is the navigation message, $C^{i}$ represents the pseudorandom code sequence, $f_{c}$ is the carrier frequency, $\Delta f$ is the frequency interval between subcarriers, $\phi_{m}^{i}$ indicates the initial phase of the $m$ th subcarrier, $g_{s}$ is the rectangular window function, and $T_{b}$ is the rectangular window function signal cycle called the sub-frame cycle.

The navigation signal contains multiple subcarrier signals and adopts multi-carrier modulation. Each subcarrier can use different pseudocodes $C^{i}$ and navigation messages $D^{i}$, and the multi-carrier is used to realize code division multiplexing through different modulation codes. Each subcarrier can use different subcarrier frequencies to superimpose and fully utilize the frequency band resources. The independent slotting control of each subcarrier can be used to realize time division multiplexing. Therefore, the navigation signal source can carry a variety of information, distinguish different carriers by different PRN codes, realize the efficient transmission of information, use the same PRN code by multi-carrier redundancy, and considerably enhance the anti-interference performance of ground augmentation signal transmission. Therefore, the signal source has great flexibility to meet the various needs in the research on and application of ground augmentation system.

\section{Results}

\subsection{Navigation ground augmentation signal-generating platform}

The design of the system hardware platform includes the controller system, the peripheral sensor circuit, the external RAM, the FLASH extension, the debugging interface, and the circuit design of the functional modules. The composition of each hardware part is introduced according to the function of the system. Figure 2 shows the basic structure of the system function hardware platform.

This study uses the LPC2129 controller, which is a 32-bit ARM7TDMI-S microprocessor produced by Philips. LPC2129 uses an embedded 256-KB high-speed flash memory and adopts the three-level pipelining technology, and the fingerprint taking, decoding, and executing are conducted simultaneously. In addition, instructions are processed parallel. Consequently, the speed of CPU operation is improved. The LPC2129 is suitable for various industrial controls due to its small volume, strong 


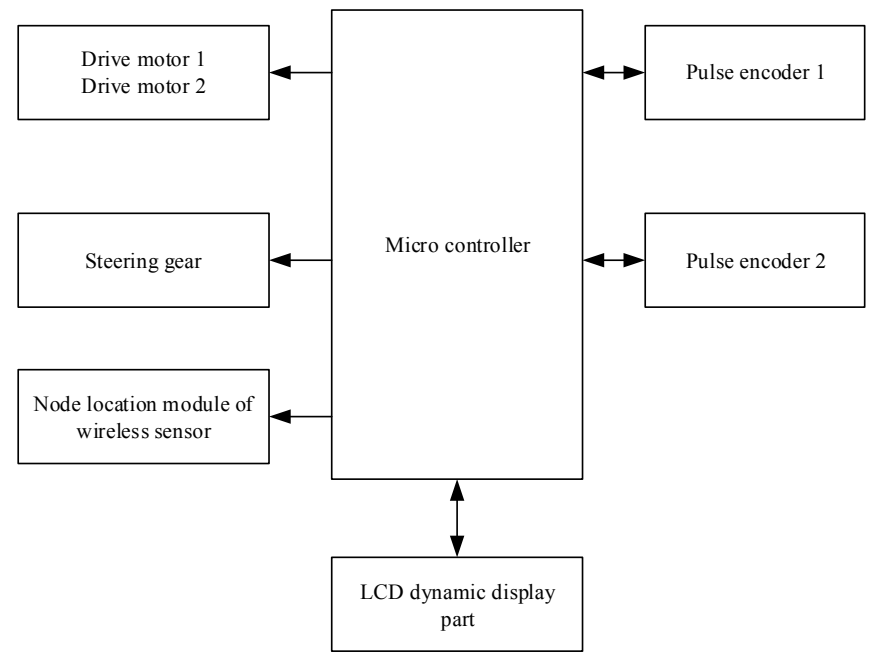

Fig. 2. Block diagram of control system hardware

anti-interference capability, and low energy consumption. It has 6-route PWM outputs, two 32-bit timing counters, and 47 general I/O ports. Thus, the LPC2129 is selected for the main controller to realize the proposed navigation ground augmentation system with stable performance. The system includes the following parts: multisensor information fusion technology, the rudder part, the sensor node location, the controller part, the driving circuit, and the LCD dynamic display part.

\subsection{Design of baseband/intermediate-frequency module}

The quality of augmented signal generated by the navigation signal augmentation platform has a significant influence on the performance of the radio navigation system. According to the designed signal source, as many as 10 subcarrier IQ signals are generated on this platform. Each subcarrier IQ has the same independent carrier generation mechanism, symbol modulation, and time slot control and can be controlled according to the demands. Multi-channel subcarrier IQ data are generated and stacked in the FPGA and then converted by the high-speed D/A module to generate intermediate-frequency IQ signals. Finally, they are mixed with the local RF carrier to the RF band.

The numerically controlled oscillator (NCO) is an important part of the software radio. It is also one of the main factors for determining the performance of the software radio in terms of generating controlled sine or cosine waves. The direct digital synthesis (DDS) technology used by the carrier NCO of this system platform has obvious advantages, such as high-frequency resolution, fast conversion, pure spectrum, continuous output phase, short stability, low-phase noise, programmable, and fully digital. DDS generally includes a reference clock, a phase accumulator, and a sine cosine lookup table. The schematic is shown in Figure 3. 


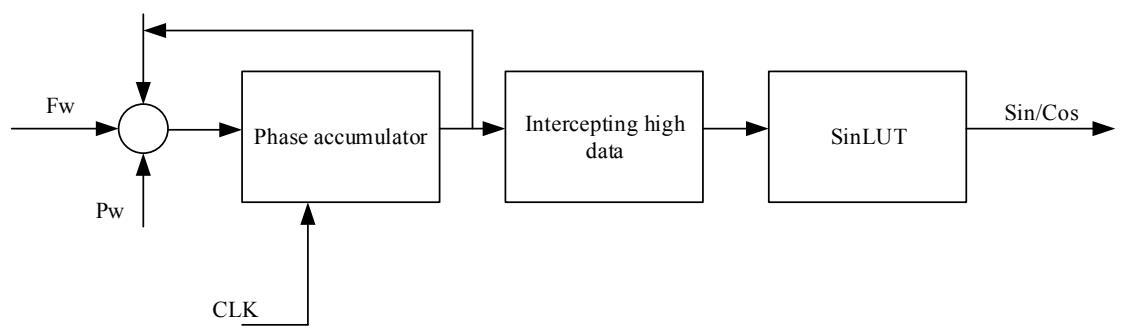

Fig. 3. Principle structure of general DDS

In Figure 4, $\mathrm{F}_{\mathrm{w}}$ represents the frequency control word of the carrier, and $\mathrm{P}_{\mathrm{w}}$ indicates the initial phase control word of the carrier. The carrier NCO is operated in accordance with the current input clock. The frequency control word is an input to the phase accumulator, which is a binary coded phase increment value. The phase accumulator includes an accumulator and a register and feedbacks the output of the register to the input end of the adder, thereby accumulating the function. The phase accumulator accumulates the frequency word incrementally under the excitation of a single clock pulse, and the phase increment adds one step length and finally outputs the step length. The output of the phase accumulator connects to the address input of the positive cosine lookup table. Under the excitation of the system clock pulse, the phase accumulator is constantly accumulating, that is, checking the table continuously. Finally, the amplitude data of the required frequency are obtained from the lookup table. The frequency control word, the system clock frequency, and the width of the phase accumulator determine the frequency of the carrier NCO output signal.

\subsection{Design of RF module and timing synchronization module}

The output of the baseband intermediate-frequency module is a digital carrier signal with modulated data; however, the RF part can only handle analog signals. Thus, the signal output from the baseband intermediate-frequency module is converted to digital mode. Through the $\mathrm{D} / \mathrm{A}$ module, the high-speed digital intermediate-frequency signal can be transformed into an analog intermediate-frequency signal. After the conversion of the D/A module, the intermediate-frequency analog IQ signal must be fused to the RF. Quadrature conversion is a primary modulation of baseband I and Q signals and is widely used in the field of communication. After the multicarrier superposition, the two-path orthogonal I $(\mathrm{t})$ and $\mathrm{Q}(\mathrm{t})$ baseband signals are multiplied by the subcarrier and the phase-shift $90^{\circ}$ carrier of the local system oscillator to form the final navigation RF signal.

When the ground augmentation system is constructed, a global reference clock is required as a reference clock of the entire network. This global reference clock is mainly realized through the $10 \mathrm{MHz}$ reference clock and the 1 PPS pulse trigger signal. The 1 PPS pulse triggering signal is important to the time of the signal generation, and all the signals must be generated by its trigger. 
In view of the difference of the batches of the hardware signal platform, the multiple augmentation signal platform is incompatible with the acquisition of the 1 PPS pulse trigger signal, which requires the system to support the delay trigger function. After the detection of the external trigger signal, the internal system can delay the entire clock cycle and start the signal generation. Consequently, the internal system can modulate according to the actual measurement of the intrinsic delay value of the equipment, so that each signal augmentation signal source after the network can begin to produce the signal at the same time. Figure 4 shows the diagram of the connection of time synchronization control module.

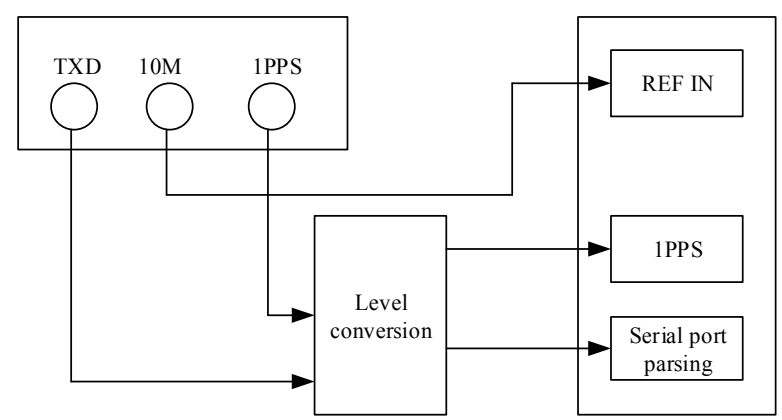

Fig. 4. Diagram of the connection of time synchronization control module

\subsection{Ground augmented receiving platform}

The ground augmented receiving platform mainly includes the down conversion processing of the received RF signals and the storage of the acquired IQ data. The signal-receiving bandwidth, the reference level of the received signal, the sampling rate, the central frequency, and other functional parameters can be set in the receiving function. On the basis of the transmitter, quadrature conversion is used to process the signal conversion. Figure 5 shows the conversion process.

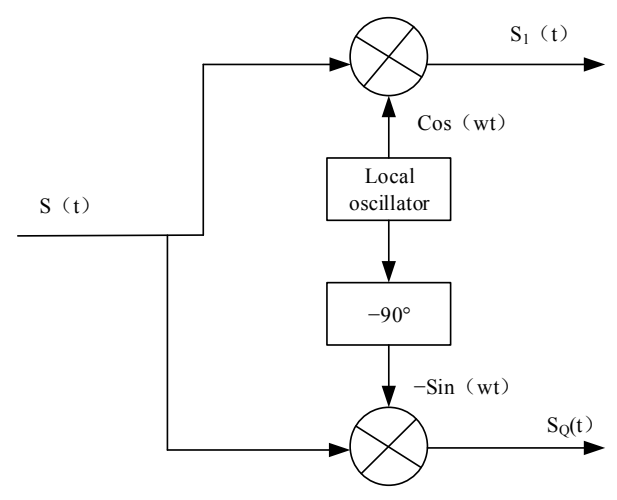

Fig. 5. Conversion process 
The acquisition and tracking verification of the enhanced signal data received by the receiving platform must be performed to verify the performance of the navigation signal augmentation platform. When the platform generates a carrier signal, it collects data through the receiving platform and stores it locally. After receiving the signal, the GPS receiver initially performs signal acquisition. Capture processing is the basis for tracking signals and demodulating navigation data. In the GPS system, the navigation message and pseudorandom code are multiplied for spread spectrum processing. The previous data signal bandwidth is broadened from $50 \mathrm{~Hz}$ to $1.023 \mathrm{MHz}(\mathrm{C} / \mathrm{A}$ code) or $10.23 \mathrm{MHz}$ (P code). Therefore, in actual GPS systems, the signal power received by the receiver antenna is extremely weak, and the signal is almost submerged in background noise. In this case, the acquisition and tracking process must be down for the weak GPS signal to be extracted from the noise to obtain the final navigation data.

Capturing is the 3D search process for the number, carrier frequency, and code phase of a satellite. The GPS receiver can only receive satellite signals in the visible range. Therefore, the satellite number must be determined for tracking and decoding. In the verification of the signal augmentation platform, the pseudocode corresponding to the satellite number can be obtained. The satellite is consistently in a high-speed motion state; thus, the Doppler frequency shift of the received signal exists, and the actual carrier frequency must be determined. GPS signal is a type of code-division multiple-access (CDMA) signal, which distinguishes different satellite signals according to different spread spectrum codes and realizes signal tracking and demodulation through the strong autocorrelation of the pseudocode sequence. The receiver's receiving signal phase is random; thus, the correct pseudocode phase and the peak value of the autocorrelation result should be obtained through the acquisition of the signal to determine the received signal that contains the pseudocode modulation signal.

A pseudocode should also be generated locally when searching for pseudocode phase. The correlation between the generated pseudorandom code and the input signal is calculated, and the phase of the generated pseudocode is continuously changed. Once the local pseudocode is aligned with the pseudocode of the input signal, a prominent correlation peak appears. If the correlation peak exceeds the set threshold, then the pseudocode phase is correct. The position of the correlation peak is particularly sensitive to phase difference. When the difference between the pseudocode of the input signal and the phase of the local pseudocode is more than one code sheet, the correlation peak will disappear quickly. Therefore, once a strong correlation peak appears, the phase difference between the local-copy pseudorandom code signal and the input signal is considered within a single chip. The capturing process is used to determine the approximate code phase value. If we want to obtain the exact code phase value, we must track the auxiliary loop. When searching on frequency, a carrier also needs to be generated locally. The locally generated carrier is multiplied with the input signal, and the frequency of the locally generated carrier is adjusted continuously. Once the local replication carrier is the same as the carrier frequency of the input signal, the high-frequency carrier component of the input signal can be removed. Multiple adjustments must be attempted for different carrier frequencies because the carrier frequency of the input signal cannot be predicted. 


\subsection{Data tracking analysis}

The carrier frequency and code phase values of the GPS signal acquisition process are rough values. The relative velocity of the antenna is constantly changing due to the continuous motion of the satellite. The carrier frequency and the pseudocode phase of the receiver antenna will change continuously due to the impact of the motion of the receiver and the random jitter of the local clock oscillator. Therefore, the signal should be tracked and the satellite navigation message should be demodulated to acquire the carrier frequency and the code phase accurately and continuously. Signal tracking has two main objectives, that is, to realize a carrier tracking loop, which can accomplish the continuous tracking of the carrier frequency and phase in the GPS signal, most of which are realized by the Costas ring; and to code tracking loop to complete the tracking of pseudocode phase, often using delayed locked loop. These two tracking loops exist in almost all GPS receivers. They are coupled and work simultaneously; however, these two rings are essentially phase-locked.

The phase-locked loop (PLL) is mainly used to lock and track the phase of the input carrier signal. It belongs to a control loop that generates and outputs periodic signals that are consistent with the input signal by changing the loop control parameters. When the output and input signal phases are the same, the PLL is locked and shows steady state characteristics. When the phase of the output signal is different from that of the input signal, the PLL is locked and has transient characteristics. If the PLL cannot enter the locked state due to interference, then it will temporarily unlock and eventually lose the navigation data. The main function of the phase detector is to compare the phases of the input and output signals and obtain the phase error signal. The essential difference between different PLLs is the difference between their phase detectors. The loop filter has a low pass characteristic, which is mainly used to filter the high-frequency component and wide-band noise in the error signal, and the control signal is used to control the voltage-controlled oscillator. Voltage-controlled oscillator is a frequency modulation oscillator, which has a linear control characteristic, and its oscillation frequency changes linearly with the change in control signal. The PLL passes the phase difference between the input and output signals continuously and changes the frequency of the output signal at a certain time. Finally, the output and input signal phases are the same.

The carrier tracking loop continuously changes the frequency of the local carrier, thereby making the locally generated carrier and the received signal carrier frequency strictly consistent. The navigation data will be turned over, which will cause the carrier of the input signal to turn in phase by $180^{\circ}$. Thus, most carrier tracking rings take the Costas ring, which is insensitive to phase reversal.

\subsection{Performance test of navigation signal augmentation platform}

After writing and compiling the program, the signal enhancement platform must test and verify the generating and receiving platforms. The functional indicators of the signals generated by the platform are verified, including the generated intermediate signals and RF signals. The verification of the receiving platform is mainly in terms 
of down conversion and storing the data part by receiving the signal generated by the platform. The process also includes capturing, tracking, and verifying data through MATLAB, which can further verify the accuracy of the generated platform signals.

When testing the signal performance of the platform, $1 \times 1.023 \mathrm{MHz}, 2 \times 1.023$ $\mathrm{MHz}, 3 \times 1.023 \mathrm{MHz}$, and three subcarriers are selected without modulation. The trigger mode of the 1 PPS signal is established. After detecting the rising edge of 1 PPS, the signal is generated after the inherent delay. When the initial phase of the three subcarriers is set at $0^{\circ}, 45^{\circ}$, and $90^{\circ}$, the baseband IQ signal is observed. Figure 6 shows the simulation results.

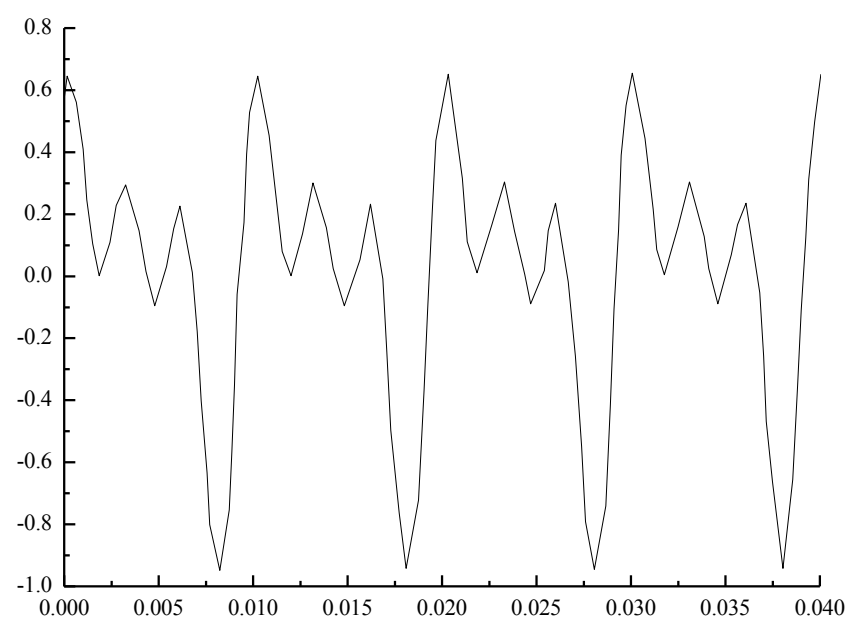

Fig. 6. Diagram of MATLAB phase superposition simulation

According to the characteristics of subcarrier generation, three single carriers of 100,200 , and $300 \mathrm{~Hz}$ are generated through MATLAB. Figure 8 shows the simulation when the initial phase is $0^{\circ}, 45^{\circ}$, and $90^{\circ}$. The comparison of the trends of peak values and waveforms shows that the initial stage of the subcarrier is effectively controlled by the platform, which is consistent with the expectation.

The data received from the receiving platform are analyzed, and the navigation message is demodulated. The analysis of data is the verification of the reception platform and the correctness of the enhanced signal generation platform. Two sets of signal augmentation platform system equipment are used, one of which launches and the other receives. To exclude other factors, such as noise, as possible, the RF OUT port of the transmitting device is directly connected to the RF IN port of the receiving device through the signal line. In the generating platform, the single carrier is set in the generation platform, the carrier frequency is $1.023 \mathrm{MHz}$, and the pseudocode rate is $1.023 \mathrm{MHz}$. The pseudocode sequence of GPS1 satellite is used, and all navigation messages are in 0101 sequence. In the receiving platform, the sampling rate is 30 $\mathrm{MS} / \mathrm{s}$ and the duration is $0.1 \mathrm{~s}$. When running the receiving platform software, the size of the received data file is $12 \mathrm{MB}$. Figure 7 shows the tracking results after setting the corresponding loop filter parameters. 


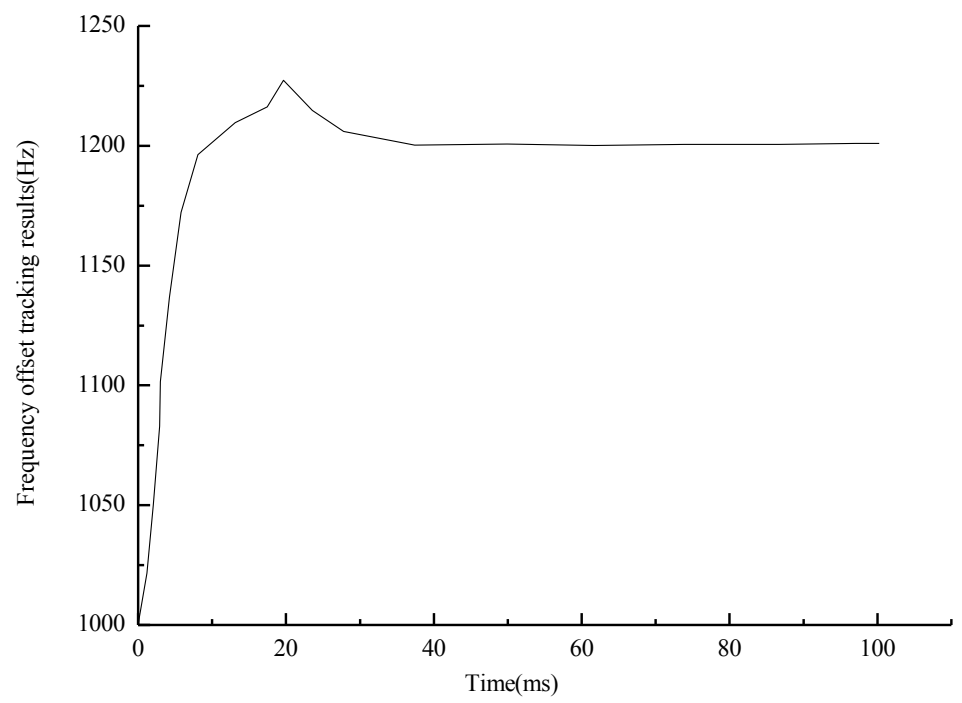

Fig. 7. Carrier tracking result

The capture and tracking analysis indicates that the navigation message and the navigation message used by the launching platform are consistent, which proves the accuracy and availability of the entire signal augmentation platform.

\section{Conclusion}

This study mainly discusses the navigation ground augmentation system based on wireless sensor. First, it reviews the research status of WSNs and the navigation system and then presents the design of the overall framework of WSN node, ground augmentation signal, and signal source. On the basis of the framework, the navigation ground augmentation signal generation platform and the ground augmented receiver platform are designed, and the performance of the navigation signal augmentation platform is tested. The test of the functions of the augmented signal-generating platform shows that the functions of the signal generated by the platform conform with the expected design. The expected navigation message data is demodulated successfully by capturing and tracking the data received on the receiving platform, and the accuracy and availability of the entire signal augmentation platform is confirmed. Therefore, the system satisfies the design requirements and can effectively improve the performance of the navigation system.

\section{References}

[1] Kang, W., Han, Y. SmartPDR: Smartphone-based pedestrian dead reckoning for indoor localization. IEEE Sensors journal, 2015, vol. 15(5), pp. 2906-2916. https://doi.org/10.1109/JSEN.2014.2382568 
[2] Fan, Q., Sun, B., Sun, Y., Zhuang, X. Performance augmentation of MEMS-based INS/UWB integration for indoor navigation applications. IEEE Sens. J, 2017, vol. 17(10), pp. 3116-3130. https://doi.org/10.1109/JSEN.2017.2689802

[3] Zeng, Y., Zhang, R., Lim, T. J. Wireless communications with unmanned aerial vehicles: opportunities and challenges. IEEE Communications Magazine, 2016, vol. 54(5), pp. 3642. https://doi.org/10.1109/MCOM.2016.7470933

[4] Dobrev, Y., Vossiek, M., Christmann, M., Bilous, I., Gulden, P. Steady Delivery: Wireless Local Positioning Systems for Tracking and Autonomous Navigation of Transport Vehicles and Mobile Robots. IEEE Microwave Magazine, 2017, vol. 18(6), pp. 26-37. https://doi.org/10.1109/MMM.2017.2711941

[5] Zhang, Z., Glaser, S. D., Bales, R. C., Conklin, M., Rice, R., Marks, D. G. Technical report: The design and evaluation of a basin - scale wireless sensor network for mountain hydrology. Water Resources Research, 2017, vol. 53(5), pp. 4487-4498. https://doi.org/10.1002/2016WR019619

[6] Aldoumani, N., Meydan, T., Dillingham, C. M., Erichsen, J. T. Enhanced Tracking System Based on Micro Inertial Measurements Unit to Measure Sensorimotor Responses in Pigeons. IEEE Sensors Journal, 2016, vol. 16(24), pp. 8847-8853. https://doi.org/10.1109/ JSEN.2016.2586540

[7] Kumar, G. A., Patil, A. K., Patil, R., Park, S. S., Chai, Y. H. A LiDAR and IMU Integrated Indoor Navigation System for UAVs and Its Application in Real-Time Pipeline Classification. Sensors, 2017, vol. 17(6), pp. 1268. https://doi.org/10.3390/s17061268

[8] Zhuang, Y., Yang, J., Li, Y., Qi, L., El-Sheimy, N. Smartphone-based indoor localization with bluetooth low energy beacons. Sensors, 2016, vol. 16(5), pp. 596. https://doi.org/10.3390/s16050596

\section{$7 \quad$ Author}

Le Le, is associate professor at Changsha Aeronautical Vocational and Technical College, Changsha, Changsha, China (lele37491@163.com). His research interests include navigation ground augmentation system.

Article submitted 27 June 2018. Resubmitted 15 July 2018. Final acceptance 30 July 2018. Final version published as submitted by the author. 